\title{
Natural Addition of Ordinals
}

\author{
Sebastian Koch \\ Johannes Gutenberg University \\ Mainz, Germany ${ }^{1}$
}

Summary. In 3 the existence of the Cantor normal form of ordinals was proven in the Mizar system [6]. In this article its uniqueness is proven and then used to formalize the natural sum of ordinals.

MSC: 03E10 68T99 03B35

Keywords: ordinal numbers; Cantor normal form; Hessenberg sum; natural sum

MML identifier: ORDINAL7, version: 8.1.09 5.57.1355

\section{INTRODUCTION}

It is well known that any ordinal number $\alpha$ can be uniquely written as

$$
\alpha=\sum_{i=1}^{k} n_{i} \omega^{\beta_{i}},
$$

where $k$ is a natural number, $n_{1}, \ldots, n_{k}$ are positive integers and $\beta_{1}>\ldots>\beta_{k}$ are ordinal numbers. This representation, usually called the Cantor Normal Form, has been formalized as the tuple $\left\langle n_{1} \omega^{\beta_{1}}, \ldots, n_{k} \omega^{\beta_{k}}\right\rangle$ in [3] and the existence of such a sequence that sums up to a given ordinal $\alpha$ has been proven in the same, but the uniqueness was omitted.

The basic proof idea for the uniqueness is well known (cf. [1], [2], 44, [5], 8] ). This article provides a variant which utilizes the additional closure of ordinals, i.e. that any ordinals $\alpha, \beta, \gamma$ with $\alpha, \beta \in \omega^{\gamma}$ also satisfy $\alpha+\beta \in \omega^{\gamma}$. Usually the

\footnotetext{
${ }^{1}$ The author is enrolled in the Johannes Gutenberg University in Mayence, Germany, mailto: skoch02@students.uni-mainz.de 
additional closure is proven using the uniqueness in the literature, but here the additional closure is proven first by using theorems from [3]. Other theorems of this article include:

- For ordinals $\alpha, \beta$ with $1 \in \alpha \in \beta$ holds $\beta+\alpha \in \alpha \beta \in \beta^{\alpha} \in \beta \uparrow \uparrow \alpha$.

- Decreasing ordinal sequences with the same range are equal.

In the last section of the article the natural sum or Hessenberg sum (cf. [2], [5]) of two ordinals $\alpha, \beta$, denoted by $\alpha \oplus \beta$, is formalized using the Cantor Normal Form. The concept of bags, as used to formalize polynomials in Mizar (cf. [7]), couldn't easily be applied in this case since there is no set of all ordinals, so it wasn't used here. The chosen definition of the natural sum turned out to be slightly sophisticated, leading to a rather long proof of its monotonicity property, while the proofs of the other shown properties are straightforward.

\section{Preliminaries}

Now we state the proposition:

(1) Let us consider a set $X$. Then $X \cap \operatorname{succ} X=X$.

Let $A$ be an increasing sequence of ordinal numbers and $a$ be an ordinal number. Let us observe that $A\lceil a$ is increasing.

Now we state the propositions:

(2) Let us consider an ordinal number $a$. Then $a+a=2 \cdot a$.

(3) Let us consider ordinal numbers $a, b$. If $1 \in a$ and $a \in b$, then $b+a \in a \cdot b$. The theorem is a consequence of (2).

(4) Let us consider an ordinal number $a$. Then $a \cdot a=a^{2}$.

Let us consider ordinal numbers $a, b$. Now we state the propositions:

(5) If $1 \in a$ and $a \in b$, then $a \cdot b \in b^{a}$. The theorem is a consequence of (4).

(6) If $1 \in a$ and $a \in b$, then $b^{a} \in b \uparrow \uparrow a$.

Let us observe that there exists a sequence of ordinal numbers which is infinite.

Now we state the propositions:

(7) Let us consider transfinite sequences $A, B$. Suppose $A^{\frown} B$ is ordinal yielding. Then

(i) $A$ is ordinal yielding, and

(ii) $B$ is ordinal yielding.

(8) Let us consider ordinal numbers $a, b$. If $a \in b$, then $b$-exponent $(a)=0$.

Let us consider ordinal numbers $a, b, c$. Now we state the propositions: 
(9) If $a \subseteq c$, then $b$-exponent $(a) \subseteq b$-exponent $(c)$.

(10) If $0 \in a$ and $1 \in b$ and $a \in b^{c}$, then $b$-exponent $(a) \in c$.

Proof: $b$-exponent $(a) \subseteq c$. $b$-exponent $(a) \neq c$.

Let us note that every sequence of ordinal numbers which is decreasing is also one-to-one. Let $A$ be a decreasing transfinite sequence and $a$ be an ordinal number. One can verify that $A\lceil a$ is decreasing.

Let $A$ be a non-decreasing transfinite sequence. One can verify that $A\lceil a$ is non-decreasing. Let $A$ be a non-increasing transfinite sequence. One can verify that $A\lceil a$ is non-increasing.

Now we state the propositions:

(11) Let us consider finite sequences $A, B$ of ordinal numbers. Then $\sum A^{\frown} B=$ $\sum A+\sum B$.

Proof: Define $\mathcal{P}$ [natural number] $\equiv$ for every finite sequences $A, B$ of ordinal numbers such that $\operatorname{dom} B=\$_{1}$ holds $\sum A \frown B=\sum A+\sum B$. $\mathcal{P}[0]$. For every natural number $n$ such that $\mathcal{P}[n]$ holds $\mathcal{P}[n+1]$. For every natural number $n, \mathcal{P}[n]$.

(12) Let us consider ordinal numbers $a, b$. Then $\sum\langle a, b\rangle=a+b$. The theorem is a consequence of (11).

Let $A$ be a non empty, non-empty, finite sequence of ordinal numbers. Let us observe that $\sum A$ is non empty.

Let $B$ be a finite sequence of ordinal numbers. Note that $\sum A \frown B$ is non empty and $\sum B^{\frown} A$ is non empty.

Now we state the propositions:

(13) Let us consider an ordinal number $a$, and a natural number $n$. Then $\sum n \longmapsto a=n \cdot a$.

(14) Let us consider a finite sequence $A$ of ordinal numbers, and an ordinal number $a$. Then $\sum A\left\lceil a \subseteq \sum A\right.$.

(15) Let us consider finite sequences $A, B$ of ordinal numbers. Suppose dom $A$ $\subseteq \operatorname{dom} B$ and for every object $a$ such that $a \in \operatorname{dom} A$ holds $A(a) \subseteq B(a)$. Then $\sum A \subseteq \sum B$.

Proof: Set $a=\operatorname{dom} A$. Consider $f_{1}$ being a sequence of ordinal numbers such that $\sum A=\operatorname{last} f_{1}$ and $\operatorname{dom} f_{1}=\operatorname{succ} \operatorname{dom} A$ and $f_{1}(0)=0$ and for every natural number $n$ such that $n \in \operatorname{dom} A$ holds $f_{1}(n+1)=f_{1}(n)+$ $A(n)$. Consider $f_{2}$ being a sequence of ordinal numbers such that $\sum B\lceil a=$ last $f_{2}$ and $\operatorname{dom} f_{2}=\operatorname{succ} \operatorname{dom}\left(B\lceil a)\right.$ and $f_{2}(0)=0$ and for every natural number $n$ such that $n \in \operatorname{dom}\left(B\lceil a)\right.$ holds $f_{2}(n+1)=f_{2}(n)+(B\lceil a)(n)$. Define $\mathcal{P}$ [natural number] $\equiv$ if $\$_{1} \in \operatorname{succ} a$, then $f_{1}\left(\$_{1}\right) \subseteq f_{2}\left(\$_{1}\right)$. For every natural number $n$ such that $\mathcal{P}[n]$ holds $\mathcal{P}[n+1]$. For every natural number $n, \mathcal{P}[n] . \sum B\left\lceil a \subseteq \sum B\right.$. 
(16) Let us consider a Cantor normal form sequence $A$ of ordinal numbers. Suppose $A \neq \emptyset$. Then there exists a Cantor normal form sequence $B$ of ordinal numbers and there exists a Cantor component ordinal number $a$ such that $A=B^{\frown}\langle a\rangle$. The theorem is a consequence of (7).

Let $A$ be a Cantor normal form sequence of ordinal numbers and $n$ be a natural number. Let us observe that $A\left\lceil n\right.$ is Cantor normal form and $A_{\lfloor n}$ is Cantor normal form and every transfinite sequence which is natural-valued is also ordinal yielding and every natural number which is limit ordinal is also zero and there exists an ordinal number which is non limit ordinal.

Let $n, m$ be natural numbers. We identify $\max (n, m)$ with $n \cup m$. We identify $\min (n, m)$ with $n \cap m$.

\section{About the Cantor Normal Form}

Now we state the proposition:

(17) Let us consider ordinal numbers $a, b$. Then $a+b=b$ if and only if $\omega \cdot a \subseteq b$. The theorem is a consequence of (2).

Let us consider a non empty, Cantor normal form sequence $A$ of ordinal numbers and an object $a$. Now we state the propositions:

(18) If $a \in \operatorname{dom} A$, then $\omega$-exponent(last $A) \subseteq \omega$-exponent $(A(a))$. The theorem is a consequence of (16).

(19) If $a \in \operatorname{dom} A$, then $\omega$-exponent $(A(a)) \subseteq \omega$-exponent $(A(0))$.

(20) Let us consider non empty, Cantor normal form sequences $A, B$ of ordinal numbers. Suppose $\omega$-exponent $(B(0)) \in \omega$-exponent(last $A$ ). Then $A^{\frown} B$ is Cantor normal form.

Proof: For every ordinal numbers $a, b$ such that $a \in b$ and $b \in \operatorname{dom}\left(A^{\frown} B\right)$ holds $\omega$-exponent $((A \frown B)(b)) \in \omega$-exponent $((A \frown B)(a))$ by [9, (20)].

(21) Let us consider decreasing sequences $A, B$ of ordinal numbers. If $\operatorname{rng} A=$ $\operatorname{rng} B$, then $A=B$.

Proof: Define $\mathcal{P}$ [natural number] $\equiv$ for every decreasing sequences $A, B$ of ordinal numbers such that len $A=\$_{1}$ and $\operatorname{rng} A=\operatorname{rng} B$ holds $A=B$. $\mathcal{P}[0]$. For every natural number $n$ such that $\mathcal{P}[n]$ holds $\mathcal{P}[n+1]$. For every natural number $n, \mathcal{P}[n]$.

Let $a$ be an ordinal number. Let us observe that $\omega^{a}$ is Cantor component.

Let $n$ be a non zero natural number. Let us note that $n \cdot \omega^{a}$ is Cantor component and every natural number which is non zero is also Cantor component.

Let $c$ be a Cantor component ordinal number. Let us observe that $\langle c\rangle$ is Cantor normal form.

Now we state the proposition: 
(22) Let us consider Cantor component ordinal numbers $c, d$.

Suppose $\omega$-exponent $(d) \in \omega$-exponent $(c)$. Then $\langle c, d\rangle$ is Cantor normal form. The theorem is a consequence of (20).

Let $a$ be a non empty ordinal number and $m$ be a non zero natural number. Note that $\left\langle\omega^{a}, m\right\rangle$ is Cantor normal form.

Let $n$ be a non zero natural number. Observe that $\left\langle n \cdot \omega^{a}, m\right\rangle$ is Cantor normal form.

Now we state the proposition:

(23) Let us consider Cantor component ordinal numbers $c, d, e$. Suppose $\omega$-exponent $(d) \in \omega$-exponent $(c)$ and $\omega$-exponent $(e) \in \omega$-exponent $(d)$. Then $\langle c, d, e\rangle$ is Cantor normal form. The theorem is a consequence of (22) and (20).

Let us consider a non empty, Cantor normal form sequence $A$ of ordinal numbers, an ordinal number $b$, and a non zero natural number $n$. Now we state the propositions:

(24) If $b \in \omega$-exponent(last $A$ ), then $A^{\frown}\left\langle n \cdot \omega^{b}\right\rangle$ is Cantor normal form. The theorem is a consequence of (20).

(25) If $\omega$-exponent $(\operatorname{last} A) \neq 0$, then $A \frown\langle n\rangle$ is Cantor normal form. The theorem is a consequence of (24).

(26) If $\omega$-exponent $(A(0)) \in b$, then $\left\langle n \cdot \omega^{b}\right\rangle \frown A$ is Cantor normal form. The theorem is a consequence of (20).

(27) Let us consider ordinal numbers $a_{1}, a_{2}, b$. If $a_{1}, a_{2} \in \omega^{b}$, then $a_{1}+a_{2} \in \omega^{b}$.

(28) Let us consider a finite sequence $A$ of ordinal numbers, and an ordinal number $b$. Suppose for every ordinal number $a$ such that $a \in \operatorname{dom} A$ holds $A(a) \in \omega^{b}$. Then $\sum A \in \omega^{b}$. The theorem can be shown by natural induction and (27).

(29) Let us consider ordinal numbers $a, b$, and a natural number $n$. If $a \in \omega^{b}$, then $n \cdot a \in \omega^{b}$. The theorem is a consequence of (28) and (13).

(30) Let us consider a finite sequence $A$ of ordinal numbers, and an ordinal number $a$. Suppose $\langle a\rangle \frown A$ is Cantor normal form. Then $\sum A \in$ $\omega^{\omega \text {-exponent }(a)}$. The theorem is a consequence of (29) and (28).

(31) Let us consider a Cantor normal form sequence $A$ of ordinal numbers. Then $\omega$-exponent $\left(\sum A\right)=\omega$-exponent $(A(0))$.

Proof: Define $\mathcal{P}$ [natural number] $\equiv$ for every Cantor normal form sequence $A$ of ordinal numbers such that len $A=\$_{1}$ holds $\omega$-exponent $\left(\sum A\right)=$ $\omega$-exponent $(A(0))$. $\mathcal{P}[0]$. For every natural number $n$ such that $\mathcal{P}[n]$ holds $\mathcal{P}[n+1]$. For every natural number $n, \mathcal{P}[n]$.

(32) Let us consider Cantor normal form sequences $A, B$ of ordinal numbers. 
If $\sum A=\sum B$, then $A=B$.

Proof: Define $\mathcal{P}$ [natural number $] \equiv$ for every Cantor normal form sequences $A, B$ of ordinal numbers such that $\operatorname{dom} A \cup \operatorname{dom} B=\$_{1}$ and $\sum A=\sum B$ holds $A=B . \mathcal{P}[0]$. For every natural number $n$ such that $\mathcal{P}[n]$ holds $\mathcal{P}[n+1]$. For every natural number $n, \mathcal{P}[n]$.

Let $A$ be a sequence of ordinal numbers and $b$ be an ordinal number. The functor $b$-exponent $(A)$ yielding a sequence of ordinal numbers is defined by

(Def. 1) $\operatorname{dom} i t=\operatorname{dom} A$ and for every object $a$ such that $a \in \operatorname{dom} A$ holds it $(a)=b$-exponent $(A(a))$.

Let $A$ be an empty sequence of ordinal numbers.

One can check that $b$-exponent $(A)$ is empty.

Let $A$ be a non empty sequence of ordinal numbers. One can verify that $b$-exponent $(A)$ is non empty. Let $A$ be a finite sequence of ordinal numbers. Let us observe that $b$-exponent $(A)$ is finite.

Let $A$ be an infinite sequence of ordinal numbers. Let us observe that $b$-exponent $(A)$ is infinite.

Now we state the propositions:

(33) Let us consider ordinal numbers $a, b$. Then $b$-exponent $(\langle a\rangle)=\langle b$-exponent $(a)\rangle$.

(34) Let us consider sequences $A, B$ of ordinal numbers, and an ordinal number $b$. Then $b$-exponent $\left(A^{\frown} B\right)=(b \text {-exponent }(A))^{\frown}(b$-exponent $(B))$.

(35) Let us consider a sequence $A$ of ordinal numbers, and ordinal numbers $b, c$. Then $b$-exponent $(A\lceil c)=(b$-exponent $(A))\lceil c$.

(36) Let us consider a finite sequence $A$ of ordinal numbers, an ordinal number $b$, and a natural number $n$. Then $b$-exponent $\left(A_{\lfloor n}\right)=(b \text {-exponent }(A))_{\lfloor n}$.

Let $A$ be a Cantor normal form sequence of ordinal numbers. Let us note that $\omega$-exponent $(A)$ is decreasing.

Now we state the propositions:

(37) Let us consider sequences $A, B$ of ordinal numbers. Suppose $A^{\wedge} B$ is Cantor normal form. Then $\operatorname{rng}(\omega$-exponent $(A))$ misses $\operatorname{rng}(\omega$-exponent $(B))$. Proof: $\operatorname{rng}(\omega$-exponent $(A)) \cap \operatorname{rng}(\omega$-exponent $(B))=\emptyset$.

(38) Let us consider a Cantor normal form sequence $A$ of ordinal numbers. Then $0 \in \operatorname{rng}(\omega$-exponent $(A))$ if and only if $A \neq \emptyset$ and $\omega$-exponent(last $A$ ) $=0$. The theorem is a consequence of (18) and (16).

Let $a, b$ be ordinal numbers. The functor $b-\mathrm{LC}(a)$ yielding an ordinal number is defined by the term

(Def. 2) $a \operatorname{div} b^{b-\operatorname{exponent}(a)}$.

Let us consider an ordinal number $a$. Now we state the propositions: 
(39) $0-\mathrm{LC}(a)=a$.

(40) $1-\mathrm{LC}(a)=a$.

(41) Let us consider an ordinal number $b$. Then $b-\mathrm{LC}(0)=0$.

(42) Let us consider ordinal numbers $a$, $b$. If $a \in b$, then $b-\mathrm{LC}(a)=a$. The theorem is a consequence of (8).

(43) Let us consider an ordinal number $b$. Then $b$-LC(1) $=1$. The theorem is a consequence of (42), (40), and (39).

(44) Let us consider ordinal numbers $a, b, c$. If $c \in b$, then $b-\mathrm{LC}\left(c \cdot b^{a}\right)=c$.

(45) Let us consider ordinal numbers $a, b$. If $1 \in b$, then $b$ - $\mathrm{LC}\left(b^{a}\right)=1$. The theorem is a consequence of (44).

Let $c$ be a Cantor component ordinal number. Observe that $\omega-\mathrm{LC}(c)$ is natural and non empty.

Now we state the proposition:

(46) Let us consider a Cantor component ordinal number $c$.

Then $c=(\omega-\mathrm{LC}(c)) \cdot \omega^{\omega-\operatorname{exponent}(c)}$. The theorem is a consequence of $(44)$.

Let $A$ be a sequence of ordinal numbers and $b$ be an ordinal number. The functor $b$ - $\mathrm{LC}(A)$ yielding a sequence of ordinal numbers is defined by

(Def. 3) $\operatorname{dom} i t=\operatorname{dom} A$ and for every object $a$ such that $a \in \operatorname{dom} A$ holds $i t(a)=b-\mathrm{LC}(A(a))$.

Let $A$ be an empty sequence of ordinal numbers. Let us observe that $b$-LC $(A)$ is empty. Let $A$ be a non empty sequence of ordinal numbers. Observe that $b-\mathrm{LC}(A)$ is non empty.

Let $A$ be a finite sequence of ordinal numbers. Let us note that $b$-LC $(A)$ is finite. Let $A$ be an infinite sequence of ordinal numbers. Let us note that $b-\mathrm{LC}(A)$ is infinite. Now we state the propositions:

(47) Let us consider ordinal numbers $a, b$. Then $b-\mathrm{LC}(\langle a\rangle)=\langle b-\mathrm{LC}(a)\rangle$.

(48) Let us consider sequences $A, B$ of ordinal numbers, and an ordinal number $b$. Then $b-\mathrm{LC}(A \frown B)=(b-\mathrm{LC}(A))^{\frown}(b-\mathrm{LC}(B))$.

(49) Let us consider a sequence $A$ of ordinal numbers, and ordinal numbers $b, c$. Then $b-\mathrm{LC}(A\lceil c)=(b-\mathrm{LC}(A))\lceil c$.

(50) Let us consider a finite sequence $A$ of ordinal numbers, an ordinal number $b$, and a natural number $n$. Then $b-\mathrm{LC}\left(A_{\lfloor n}\right)=(b-\mathrm{LC}(A))_{\lfloor n}$.

Let $A$ be a Cantor normal form sequence of ordinal numbers and $a$ be an object. Note that $(\omega-\mathrm{LC}(A))(a)$ is natural and $\omega-\mathrm{LC}(A)$ is natural-valued and non-empty.

Let us consider a Cantor normal form sequence $A$ of ordinal numbers and an object $a$. Now we state the propositions: 
(51) If $a \in \operatorname{dom} A$, then $A(a)=(\omega-\operatorname{LC}(A(a))) \cdot \omega^{\omega-\operatorname{exponent}(A(a))}$. The theorem is a consequence of (46).

(52) If $a \in \operatorname{dom} A$, then $A(a)=(\omega-\operatorname{LC}(A))(a) \cdot \omega^{(\omega-\operatorname{exponent}(A))(a)}$. The theorem is a consequence of (51).

(53) Let us consider a decreasing sequence $A$ of ordinal numbers, and a naturalvalued, non-empty sequence $B$ of ordinal numbers. Suppose $\operatorname{dom} A=$ $\operatorname{dom} B$. Then there exists a Cantor normal form sequence $C$ of ordinal numbers such that

(i) $\omega$-exponent $(C)=A$, and

(ii) $\omega-\mathrm{LC}(C)=B$.

Proof: Define $\mathcal{F}$ (ordinal number) $=B\left(\$_{1}\right) \cdot \omega^{A\left(\$_{1}\right)}$. Consider $C$ being a sequence of ordinal numbers such that $\operatorname{dom} C=\operatorname{dom} A$ and for every ordinal number $a$ such that $a \in \operatorname{dom} A$ holds $C(a)=\mathcal{F}(a)$.

(54) Let us consider Cantor normal form sequences $A, B$ of ordinal numbers. Suppose $\omega$-exponent $(A)=\omega$-exponent $(B)$ and $\omega-\operatorname{LC}(A)=\omega-\operatorname{LC}(B)$. Then $A=B$. The theorem is a consequence of (52).

Let $a$ be an ordinal number. The functor $\operatorname{CNF}(a)$ yielding a Cantor normal form sequence of ordinal numbers is defined by

(Def. 4) $\sum i t=a$.

Note that $\sum \operatorname{CNF}(a)$ reduces to $a$. Let $A$ be a Cantor normal form sequence of ordinal numbers. One can check that $\operatorname{CNF}\left(\sum A\right)$ reduces to $A$.

Now we state the proposition:

(55) $\operatorname{CNF}(\emptyset)=\emptyset$.

Let $a$ be an empty ordinal number. Note that $\operatorname{CNF}(a)$ is empty.

Let $a$ be a non empty ordinal number. Note that $\operatorname{CNF}(a)$ is non empty.

Now we state the propositions:

(56) Let us consider an ordinal number $a$, and a non zero natural number $n$. Then $\operatorname{CNF}\left(n \cdot \omega^{a}\right)=\left\langle n \cdot \omega^{a}\right\rangle$.

(57) Let us consider a Cantor component ordinal number $a$. Then $\operatorname{CNF}(a)=$ $\langle a\rangle$.

(58) Let us consider a non zero natural number $n$. Then $\operatorname{CNF}(n)=\langle n\rangle$.

(59) Let us consider a non empty ordinal number $a$, and non zero natural numbers $n, m$. Then $\operatorname{CNF}\left(n \cdot \omega^{a}+m\right)=\left\langle n \cdot \omega^{a}, m\right\rangle$. The theorem is a consequence of (12).

(60) Let us consider a non empty ordinal number $a$, an ordinal number $b$, and a non zero natural number $n$. Suppose $b \in \omega$-exponent(last $\operatorname{CNF}(a)$ ). Then $\operatorname{CNF}\left(a+n \cdot \omega^{b}\right)=\operatorname{CNF}(a) \frown\left\langle n \cdot \omega^{b}\right\rangle$. The theorem is a consequence of (24). 
(61) Let us consider a non empty ordinal number $a$, and a non zero natural number $n$. Suppose $\omega$-exponent $($ last $\operatorname{CNF}(a)) \neq 0$. Then $\operatorname{CNF}(a+n)=$ $\operatorname{CNF}(a) \frown\langle n\rangle$. The theorem is a consequence of (60).

(62) Let us consider a non empty ordinal number $a$, an ordinal number $b$, and a non zero natural number $n$. Suppose $\omega$-exponent $((\operatorname{CNF}(a))(0)) \in b$. Then $\operatorname{CNF}\left(n \cdot \omega^{b}+a\right)=\left\langle n \cdot \omega^{b}\right\rangle \frown \operatorname{CNF}(a)$. The theorem is a consequence of (26).

\section{Natural Addition of Ordinals}

Let $a, b$ be ordinal numbers. The functor $a \oplus b$ yielding an ordinal number is defined by

(Def. 5) there exists a Cantor normal form sequence $C$ of ordinal numbers such that it $=\sum C$ and $\operatorname{rng}(\omega$-exponent $(C))=\operatorname{rng}(\omega$-exponent $(\operatorname{CNF}(a))) \cup$ $\operatorname{rng}(\omega$-exponent $(\operatorname{CNF}(b)))$ and for every object $d$ such that $d \in \operatorname{dom} C$ holds:

if $\omega$-exponent $(C(d)) \in \operatorname{rng}(\omega$-exponent $(\mathrm{CNF}(a))) \backslash \operatorname{rng}(\omega$-exponent $(\mathrm{CNF}$ $(b)))$, then $\omega-\mathrm{LC}(C(d))=(\omega-\mathrm{LC}(\mathrm{CNF}(a)))\left(\left((\omega-\operatorname{exponent}(\operatorname{CNF}(a)))^{-1}\right)\right.$ $(\omega$-exponent $(C(d))))$ and

if $\omega$-exponent $(C(d)) \in \operatorname{rng}(\omega$-exponent $(\mathrm{CNF}(b))) \backslash \operatorname{rng}(\omega$-exponent $(\mathrm{CNF}$ $(a)))$, then $\omega-\mathrm{LC}(C(d))=(\omega-\mathrm{LC}(\mathrm{CNF}(b)))\left(\left((\omega-\operatorname{exponent}(\mathrm{CNF}(b)))^{-1}\right)\right.$ $(\omega$-exponent $(C(d))))$ and

if $\omega$-exponent $(C(d)) \in \operatorname{rng}(\omega$-exponent $(\mathrm{CNF}(a))) \cap \operatorname{rng}(\omega$-exponent $(\mathrm{CNF}$ $(b)))$, then $\omega-\mathrm{LC}(C(d))=(\omega-\mathrm{LC}(\mathrm{CNF}(a)))\left(\left((\omega-\operatorname{exponent}(\operatorname{CNF}(a)))^{-1}\right)\right.$ $(\omega$-exponent $(C(d))))+(\omega-\mathrm{LC}(\mathrm{CNF}(b)))\left(\left((\omega \text {-exponent }(\mathrm{CNF}(b)))^{-1}\right)\right.$ $(\omega$-exponent $(C(d))))$.

One can verify that the functor is commutative.

Let us consider ordinal numbers $a, b$. Now we state the propositions:

(63) $\operatorname{rng}(\omega$-exponent $(\mathrm{CNF}(a \oplus b)))=$ $\operatorname{rng}(\omega$-exponent $(\mathrm{CNF}(a))) \cup \operatorname{rng}(\omega$-exponent $(\mathrm{CNF}(b)))$.

(64) $\operatorname{dom}(\operatorname{CNF}(a)) \subseteq \operatorname{dom}(\operatorname{CNF}(a \oplus b))$. The theorem is a consequence of (63).

Let us consider ordinal numbers $a, b$ and an object $d$. Now we state the propositions:

(65) Suppose $d \in \operatorname{dom}(\operatorname{CNF}(a \oplus b))$ and $\omega$-exponent $((\operatorname{CNF}(a \oplus b))(d)) \in$ $\operatorname{rng}(\omega$-exponent $(\mathrm{CNF}(a))) \backslash \operatorname{rng}(\omega$-exponent $(\mathrm{CNF}(b)))$. Then $\omega$-LC $((\mathrm{CNF}$ $(a \oplus b))(d))=(\omega-\operatorname{LC}(\operatorname{CNF}(a)))\left(\left((\omega \text {-exponent }(\operatorname{CNF}(a)))^{-1}\right)(\omega\right.$-exponent $((\mathrm{CNF}(a \oplus b))(d))))$. 
(66) Suppose $d \in \operatorname{dom}(\operatorname{CNF}(a \oplus b))$ and $\omega$-exponent $((\operatorname{CNF}(a \oplus b))(d)) \in$ $\operatorname{rng}(\omega$-exponent $(\mathrm{CNF}(b))) \backslash \operatorname{rng}(\omega$-exponent $(\mathrm{CNF}(a)))$. Then $\omega$-LC $((\mathrm{CNF}$ $(a \oplus b))(d))=(\omega-\mathrm{LC}(\mathrm{CNF}(b)))\left(\left((\omega \text {-exponent }(\mathrm{CNF}(b)))^{-1}\right)(\omega\right.$-exponent $((\mathrm{CNF}(a \oplus b))(d))))$.

(67) Suppose $d \in \operatorname{dom}(\operatorname{CNF}(a \oplus b))$ and $\omega$-exponent $((\operatorname{CNF}(a \oplus b))(d)) \in$ $\operatorname{rng}(\omega$-exponent $(\mathrm{CNF}(a))) \cap \operatorname{rng}(\omega$-exponent $(\mathrm{CNF}(b)))$. Then $\omega-\mathrm{LC}((\mathrm{CNF}$ $(a \oplus b))(d))=(\omega-\operatorname{LC}(\operatorname{CNF}(a)))\left(\left((\omega \text {-exponent }(\operatorname{CNF}(a)))^{-1}\right)(\omega\right.$-exponent $((\mathrm{CNF}(a \oplus b))(d))))+(\omega-\mathrm{LC}(\mathrm{CNF}(b)))\left(\left((\omega \text {-exponent }(\mathrm{CNF}(b)))^{-1}\right)\right.$ $(\omega$-exponent $((\mathrm{CNF}(a \oplus b))(d))))$.

(68) Let us consider ordinal numbers $a, b, c$. Then $(a \oplus b) \oplus c=a \oplus(b \oplus c)$.

(69) Let us consider an ordinal number $a$. Then $a \oplus 0=a$.

(70) Let us consider ordinal numbers $a, b$, and a natural number $n$. Suppose $\omega$-exponent $(a) \subseteq b$. Then $n \cdot \omega^{b} \oplus a=n \cdot \omega^{b}+a$. The theorem is a consequence of (31), (69), (56), (33), (21), (47), (44), (51), and (52).

(71) Let us consider finite sequences $A, B$ of ordinal numbers. Suppose $A^{\frown} B$ is Cantor normal form. Then $\sum A \oplus \sum B=\sum A+\sum B$.

Proof: Define $\mathcal{P}$ [natural number] $\equiv$ for every finite sequences $A, B$ of ordinal numbers such that len $A=\$_{1}$ and $A^{\frown} B$ is Cantor normal form holds $\sum A \oplus \sum B=\sum A+\sum B . \mathcal{P}[0]$. For every natural number $n$ such that $\mathcal{P}[n]$ holds $\mathcal{P}[n+1]$. For every natural number $n, \mathcal{P}[n]$.

(72) Let us consider ordinal numbers $a, b$. Suppose if $a \neq 0$, then $\omega$-exponent $(b)$ $\in \omega$-exponent(last $\operatorname{CNF}(a))$. Then $a \oplus b=a+b$. The theorem is a consequence of (69), (31), (20), and (71).

(73) Let us consider ordinal numbers $a, b$, and a natural number $n$. Suppose if $a \neq 0$, then $b \subseteq \omega$-exponent(last $\operatorname{CNF}(a))$. Then $a \oplus n \cdot \omega^{b}=a+n \cdot \omega^{b}$. The theorem is a consequence of (69), (16), (70), (11), (71), (68), and (12).

(74) Let us consider an ordinal number $a$, and natural numbers $n, m$. Then $n \cdot \omega^{a} \oplus m \cdot \omega^{a}=(n+m) \cdot \omega^{a}$. The theorem is a consequence of (69), (56), and (73).

(75) Let us consider an ordinal number $a$, and a natural number $n$. Then $a \oplus n=a+n$. The theorem is a consequence of (73).

(76) Let us consider natural numbers $n, m$. Then $n \oplus m=n+m$. The theorem is a consequence of (75).

Let $n, m$ be natural numbers. We identify $n+m$ with $n \oplus m$. Now we state the propositions:

(77) Let us consider an ordinal number $a$. Then $a \oplus 1=\operatorname{succ} a$. The theorem is a consequence of $(75)$. 
(78) Let us consider ordinal numbers $a, b$. Then $a \oplus \operatorname{succ} b=\operatorname{succ}(a \oplus b)$. The theorem is a consequence of (77) and (68).

Let $a$ be an empty ordinal number. Let us note that $a \oplus a$ is empty.

Let $a$ be a non empty ordinal number and $b$ be an ordinal number. Let us note that $a \oplus b$ is non empty. Now we state the proposition:

(79) Let us consider an ordinal number $a$. Then $a$ is limit ordinal if and only if $0 \notin \operatorname{rng}(\omega$-exponent $(\operatorname{CNF}(a)))$. The theorem is a consequence of (16), (46), (38), (77), (58), (33), and (8).

Let $a, b$ be limit ordinal ordinal numbers. Let us note that $a \oplus b$ is limit ordinal. Let $a$ be an ordinal number and $b$ be a non limit ordinal ordinal number. One can check that $a \oplus b$ is non limit ordinal.

Now we state the propositions:

(80) Let us consider ordinal numbers $a, b$, and a non zero natural number $n$. Suppose $n \cdot \omega^{b} \subseteq a$ and $a \in(n+1) \cdot \omega^{b}$. Then $(\operatorname{CNF}(a))(0)=n \cdot \omega^{b}$.

Proof: Consider $a_{0}$ being a Cantor component ordinal number, $A_{0}$ being a Cantor normal form sequence of ordinal numbers such that $\operatorname{CNF}(a)=$ $\left\langle a_{0}\right\rangle^{\frown} A_{0} . b \subseteq \omega$-exponent $(a) \subseteq b$. Reconsider $m=\omega-\operatorname{LC}((\operatorname{CNF}(a))(0))$ as a natural number. $(\operatorname{CNF}(a))(0)=m \cdot \omega^{b} \cdot m=n$.

(81) Let us consider ordinal numbers $a, b$. Suppose $\operatorname{rng}(\omega$-exponent $(\operatorname{CNF}(a)))$ $=\operatorname{rng}(\omega$-exponent $(\mathrm{CNF}(b)))$. Let us consider an ordinal number $c$. Suppose $c \in \operatorname{dom}(\mathrm{CNF}(a))$. Then $(\omega-\mathrm{LC}(\mathrm{CNF}(a \oplus b)))(c)=(\omega-\mathrm{LC}(\mathrm{CNF}(a)))(c)+$ $(\omega-\mathrm{LC}(\mathrm{CNF}(b)))(c)$. The theorem is a consequence of $(21)$.

Let us consider ordinal numbers $a, b$. Now we state the propositions:

(i) if $\omega$-exponent $((\operatorname{CNF}(a \oplus b))(0)) \in \operatorname{rng}(\omega$-exponent $(\operatorname{CNF}(a)))$, then $\omega$-exponent $((\operatorname{CNF}(a \oplus b))(0))=(\omega$-exponent $(\operatorname{CNF}(a)))(0)$, and

(ii) if $\omega$-exponent $((\operatorname{CNF}(a \oplus b))(0)) \in \operatorname{rng}(\omega$-exponent $(\operatorname{CNF}(b)))$, then $\omega$-exponent $((\mathrm{CNF}(a \oplus b))(0))=(\omega$-exponent $(\mathrm{CNF}(b)))(0)$.

Proof: Set $E_{1}=\omega$-exponent $(\operatorname{CNF}(a))$. Set $E_{2}=\omega$-exponent $(\operatorname{CNF}(b))$. Set $C_{0}=\operatorname{CNF}(a \oplus b) \cdot \operatorname{rng}\left(\omega\right.$-exponent $\left.\left(C_{0}\right)\right)=\operatorname{rng} E_{1} \cup \operatorname{rng} E_{2}$. Consider $x$ being an object such that $x \in \operatorname{dom} E_{2}$ and $E_{2}(x)=\omega$-exponent $\left(C_{0}(0)\right)$. $x=0$.

(i) if $\omega$-exponent $((\operatorname{CNF}(a \oplus b))(0)) \in \operatorname{rng}(\omega$-exponent $(\mathrm{CNF}(a))) \backslash \operatorname{rng}$ $(\omega$-exponent $(\operatorname{CNF}(b)))$, then $(\operatorname{CNF}(a \oplus b))(0)=(\operatorname{CNF}(a))(0)$, and

(ii) if $\omega$-exponent $((\operatorname{CNF}(a \oplus b))(0)) \in \operatorname{rng}(\omega$-exponent $(\operatorname{CNF}(b))) \backslash \operatorname{rng}$ $(\omega$-exponent $(\operatorname{CNF}(a)))$, then $(\operatorname{CNF}(a \oplus b))(0)=(\operatorname{CNF}(b))(0)$, and

(iii) if $\omega$-exponent $((\operatorname{CNF}(a \oplus b))(0)) \in \operatorname{rng}(\omega$-exponent $(\operatorname{CNF}(a))) \cap \operatorname{rng}$ $(\omega$-exponent $(\operatorname{CNF}(b)))$, then $(\operatorname{CNF}(a \oplus b))(0)=(\operatorname{CNF}(a))(0)+(\operatorname{CNF}$ $(b))(0)$. 
The theorem is a consequence of (82), (51), and (52).

Let us consider ordinal numbers $a, b$ and an object $x$. Now we state the propositions:

Proof: Set $E_{1}=\omega$-exponent $(\operatorname{CNF}(a))$. Set $E_{2}=\omega$-exponent $(\operatorname{CNF}(b))$. Set $C_{0}=\operatorname{CNF}(a \oplus b)$. Define $\mathcal{P}$ [ordinal number $] \equiv\left(\omega\right.$-exponent $\left.\left(C_{0}\right)\right)\left(\$_{1}\right) \in$ $E_{1}\left(\$_{1}\right)$. There exists an ordinal number $z$ such that $\mathcal{P}[z]$. Consider $y$ being an ordinal number such that $\mathcal{P}[y]$ and for every ordinal number $z$ such that $\mathcal{P}[z]$ holds $y \subseteq z \cdot \operatorname{rng}\left(\omega\right.$-exponent $\left.\left(C_{0}\right)\right)=\operatorname{rng} E_{1} \cup \operatorname{rng} E_{2}$. Consider $z$ being an object such that $z \in \operatorname{dom}\left(\omega\right.$-exponent $\left.\left(C_{0}\right)\right)$ and $\left(\omega\right.$-exponent $\left.\left(C_{0}\right)\right)(z)=$ $E_{1}(y) . z \in y$.

(85) $(\mathrm{CNF}(a))(x) \subseteq(\mathrm{CNF}(a \oplus b))(x)$.

Proof: Set $E_{1}=\omega$-exponent $(\operatorname{CNF}(a))$. Set $E_{2}=\omega$-exponent $(\operatorname{CNF}(b))$. Set $L_{1}=\omega-\operatorname{LC}(\operatorname{CNF}(a))$. Set $L_{2}=\omega-\operatorname{LC}(\operatorname{CNF}(b))$. Set $C_{0}=\operatorname{CNF}(a \oplus b)$.

Consider $C$ being a Cantor normal form sequence of ordinal numbers such that $a \oplus b=\sum C$ and $\operatorname{rng}(\omega$-exponent $(C))=\operatorname{rng} E_{1} \cup \operatorname{rng} E_{2}$ and for every object $d$ such that $d \in \operatorname{dom} C$ holds if $\omega$-exponent $(C(d)) \in$ $\operatorname{rng} E_{1} \backslash \operatorname{rng} E_{2}$, then $\omega-\mathrm{LC}(C(d))=L_{1}\left(\left(E_{1}{ }^{-1}\right)(\omega-\operatorname{exponent}(C(d)))\right)$ and if $\omega$-exponent $(C(d)) \in \operatorname{rng} E_{2} \backslash \operatorname{rng} E_{1}$, then $\omega-\operatorname{LC}(C(d))=L_{2}\left(\left(E_{2}{ }^{-1}\right)\right.$ $(\omega$-exponent $(C(d))))$ and if $\omega$-exponent $(C(d)) \in \operatorname{rng} E_{1} \cap \operatorname{rng} E_{2}$, then $\omega-\mathrm{LC}(C(d))=L_{1}\left(\left(E_{1}^{-1}\right)(\omega\right.$-exponent $\left.(C(d)))\right)+L_{2}\left(\left(E_{2}{ }^{-1}\right)(\omega\right.$-exponent $(C(d))))$.

$$
\operatorname{dom}(\mathrm{CNF}(a)) \subseteq \operatorname{dom}(\mathrm{CNF}(a \oplus b)) . C_{0}(x)=\left(\omega-\mathrm{LC}\left(C_{0}\right)\right)(x) .
$$

$\omega^{\left(\omega-\operatorname{exponent}\left(C_{0}\right)\right)(x)} \cdot(\operatorname{CNF}(a))(x)=L_{1}(x) \cdot \omega^{E_{1}(x)} \cdot E_{1}(x)=(\omega$-exponent $\left.\left(C_{0}\right)\right)(x)$.

Let us consider ordinal numbers $a, b$. Now we state the propositions:

(86) $a \subseteq a \oplus b$. The theorem is a consequence of (64), (85), and (15).

(87) $\omega$-exponent $(a \oplus b)=(\omega$-exponent $(a)) \cup(\omega$-exponent $(b))$. The theorem is a consequence of (9), (86), (63), (82), and (31).

(88) Let us consider ordinal numbers $a, b$, $c$. If $a, b \in \omega^{c}$, then $a \oplus b \in \omega^{c}$. The theorem is a consequence of (69), (10), and (87).

The scheme OrdinalCNFIndA deals with a unary predicate $\mathcal{P}$ and states that

(Sch. 1) For every non empty ordinal number $a, \mathcal{P}[a]$ provided

- for every ordinal number $a$ and for every non zero natural number $n$, $\mathcal{P}\left[n \cdot \omega^{a}\right]$ and 
- for every ordinal number $a$ and for every non empty ordinal number $b$ and for every non zero natural number $n$ such that $\mathcal{P}[b]$ and $a \notin$ $\operatorname{rng}(\omega$-exponent $(\mathrm{CNF}(b)))$ holds $\mathcal{P}\left[b \oplus n \cdot \omega^{a}\right]$.

The scheme OrdinalCNFIndB deals with a unary predicate $\mathcal{P}$ and states that

(Sch. 2) For every non empty ordinal number $a, \mathcal{P}[a]$

provided

- for every ordinal number $a, \mathcal{P}\left[\omega^{a}\right]$ and

- for every ordinal number $a$ and for every non zero natural number $n$ such that $\mathcal{P}\left[n \cdot \omega^{a}\right]$ holds $\mathcal{P}\left[(n+1) \cdot \omega^{a}\right]$ and

- for every ordinal number $a$ and for every non empty ordinal number $b$ and for every non zero natural number $n$ such that $\mathcal{P}[b]$ and $a \notin$ $\operatorname{rng}(\omega$-exponent $(\mathrm{CNF}(b)))$ holds $\mathcal{P}\left[b \oplus n \cdot \omega^{a}\right]$.

The scheme OrdinalCNFIndC deals with a unary predicate $\mathcal{P}$ and states that

(Sch. 3) For every non empty ordinal number $a, \mathcal{P}[a]$

provided

- for every ordinal number $a, \mathcal{P}\left[\omega^{a}\right]$ and

- for every ordinal number $a$ and for every non empty ordinal number $b$ such that $\mathcal{P}[b]$ holds $\mathcal{P}\left[b \oplus \omega^{a}\right]$.

Now we state the propositions:

(89) Let us consider ordinal numbers $a, b$.

Suppose $\omega$-exponent $(a) \in \omega$-exponent $(b)$. Then $a \in \omega^{\omega-\operatorname{exponent}(b)}$.

Proof: Define $\mathcal{P}$ [non empty ordinal number $] \equiv$ for every ordinal number $b$ such that $\omega$-exponent $\left(\$_{1}\right) \in \omega$-exponent $(b)$ holds $\$_{1} \in \omega^{\omega-\operatorname{exponent}(b)}$. For every ordinal number $c$ and for every non zero natural number $n$, $\mathcal{P}\left[n \cdot \omega^{c}\right]$. For every ordinal number $c$ and for every non empty ordinal number $d$ and for every non zero natural number $n$ such that $\mathcal{P}[d]$ and $c \notin \operatorname{rng}(\omega$-exponent $(\operatorname{CNF}(d)))$ holds $\mathcal{P}\left[d \oplus n \cdot \omega^{c}\right]$. For every non empty ordinal number $a, \mathcal{P}[a]$.

(90) Let us consider non empty ordinal numbers $a, b$. Then $\omega \cdot a \subseteq b$ if and only if $\omega$-exponent $(a) \in \omega$-exponent $(b)$. The theorem is a consequence of (89) and (29).

Let us consider ordinal numbers $a, b$. Now we state the propositions: 
(91) If $\omega$-exponent $(a) \in \omega$-exponent $(b)$, then $b-a=b$. The theorem is a consequence of (90), (17), and (89).

(92) $a+b \subseteq a \oplus b$.

Proof: Define $\mathcal{P}$ [natural number $] \equiv$ for every non empty ordinal numbers $a, b$ such that len $\operatorname{CNF}(a)=\$_{1}$ holds $a+b \subseteq a \oplus b . \mathcal{P}[1]$. For every non zero natural number $n$ such that $\mathcal{P}[n]$ holds $\mathcal{P}[n+1]$. For every non zero natural number $n, \mathcal{P}[n]$.

Let us consider ordinal numbers $a, b, c$. Now we state the propositions:

(93) If $a \oplus b=a \oplus c$, then $b=c$.

Proof: Set $E_{2}=\omega$-exponent $(\mathrm{CNF}(b))$. Set $E_{3}=\omega$-exponent $(\mathrm{CNF}(c))$. Set $L_{2}=\omega-\mathrm{LC}(\mathrm{CNF}(b))$. Set $L_{3}=\omega-\mathrm{LC}(\mathrm{CNF}(c)) \cdot \operatorname{rng} E_{2}=\operatorname{rng} E_{3}$. $E_{2}=E_{3}$. For every object $x$ such that $x \in \operatorname{dom} L_{2}$ holds $L_{2}(x)=L_{3}(x)$. $\sum \mathrm{CNF}(b)=\sum \mathrm{CNF}(c)$.

(94) If $b \in c$, then $a \oplus b \in a \oplus c$. The theorem is a consequence of (69), (11), (71), and (68).

(95) If $b \subseteq c$, then $a \oplus b \subseteq a \oplus c$. The theorem is a consequence of (94).

\section{REFERENCES}

[1] Alexander Abian. The theory of sets and transfinite arithmetic. Saunders mathematics books. Saunders, Philadelphia [u.a.], 1965.

[2] Heinz Bachmann. Transfinite Zahlen. Ergebnisse der Mathematik und ihrer Grenzgebiete, (1). Springer, Berlin [u.a.], 2., neubearb. aufl. edition, 1967.

[3] Grzegorz Bancerek. Epsilon numbers and Cantor normal form. Formalized Mathematics, 17(4):249-256, 2009. doi $10.2478 / \mathrm{v} 10037-009-0032-8$

[4] Georg Cantor. Beiträge zur begründung der transfiniten mengenlehre. Mathematische Annalen, 49(2):207-246, 1897.

[5] Oliver Deiser. Einführung in die Mengenlehre: die Mengenlehre Georg Cantors und ihre Axiomatisierung durch Ernst Zermelo. Springer, Berlin [u.a.], 2., verb. und erw. aufl. edition, 2004. ISBN 3-540-20401-6.

[6] Adam Grabowski, Artur Korniłowicz, and Adam Naumowicz. Four decades of Mizar. Journal of Automated Reasoning, 55(3):191-198, 2015. doi:10.1007/s10817-015-9345-1

[7] Piotr Rudnicki and Andrzej Trybulec. Multivariate polynomials with arbitrary number of variables Formalized Mathematics, 9(1):95-110, 2001.

[8] Wacław Sierpiński. Cardinal and ordinal numbers. Polska Akademia Nauk. Monografie matematyczne, (34) (in Polish). PWN, Warszawa, 2. ed., rev edition, 1965.

[9] Tetsuya Tsunetou, Grzegorz Bancerek, and Yatsuka Nakamura. Zero-based finite sequences Formalized Mathematics, 9(4):825-829, 2001. 